hep-th/0603088

\title{
Stabilizing Moduli with a Positive Cosmological Constant in Heterotic M-Theory
}

\author{
Volker Braun and Burt A. Ovrut \\ Department of Physics, University of Pennsylvania \\ Philadelphia, PA 19104-6396
}

\begin{abstract}
It is shown that strongly coupled heterotic $M$-theory with anti-five-branes in the $S^{1} / \mathbb{Z}_{2}$ bulk space can have meta-stable vacua which break $N=1$ supersymmetry and have a small, positive cosmological constant. This is demonstrated for the "minimal" heterotic standard model. This vacuum has the exact MSSM matter spectrum in the observable sector, a trivial hidden sector vector bundle and both five-branes and antifive-branes in the bulk space. The Kähler moduli for which the cosmological constant has phenomenologically acceptable values are shown to also render the observable sector vector bundle slope-stable. A corollary of this result is that strongly coupled $M$-theory vacua with only five-branes in the $S^{1} / \mathbb{Z}_{2}$ interval may have stabilized moduli, but at a supersymmetry preserving minimum with a large, negative cosmological constant.
\end{abstract}




\section{Introduction}

In a series of papers, vacuum states of the $E_{8} \times E_{8}$ heterotic superstring were presented whose observable sectors have the matter spectrum of the minimal supersymmetric standard model (MSSM) with the addition of one extra pair of Higgs-Higgs conjugate fields [1, 2, 3]. Subsequently, it was shown [4, 5] that a subclass of these theories have exactly the matter content of the MSSM. Since the observable sector matter spectra are realistic, these ground states are called "heterotic standard models". The vacuum with exactly the MSSM matter spectrum is called the "minimal" heterotic standard model. In the last year similar observable sectors were found using orbifolds [6] or geometrically with a supersymmetric hidden sector [7, 8]. Other approaches can be found in [9, 10, 11, 12, 13, 14]

The vacua [1, 2, 3, 4] are constructed by compactifying the $E_{8} \times E_{8}$ heterotic string on a torus-fibered Calabi-Yau threefold whose fundamental group is $\mathbb{Z}_{3} \times \mathbb{Z}_{3}[15]$. The observable sector contains a holomorphic vector bundle $V$ with structure group $S U(4)$. This bundle was proven to be slope-stable for the two Higgs pair vacua in 16] and for the minimal heterotic standard model in [17]. Hence, in all cases the observable sector admits a gauge connection satisfying the hermitian Yang-Mills equations. The $S U(4)$ structure group breaks $E_{8}$ down to $\operatorname{Spin}(10)$. The discovery of non-vanishing neutrino masses requires that realistic supersymmetric theories contain right-handed neutrinos [18, 19]. It is well-known that the 16 representation of $\operatorname{Spin}(10)$ is composed of an entire family of quarks/leptons, including a right-handed neutrino. For this reason, an $S U(4)$ vector bundle was chosen for the observable sector of heterotic standard models. A formalism for computing the number of vector bundle moduli was presented in [20] and applied to the observable sector bundles. For example, the number of vector bundle moduli in the observable sector of the minimal heterotic standard model is thirteen. The Spin(10) gauge group is then broken by $\mathbb{Z}_{3} \times \mathbb{Z}_{3}$ Wilson lines. Since $\mathbb{Z}_{3} \times \mathbb{Z}_{3}$ is Abelian, the low energy theory consists of the standard model gauge group, $S U(3)_{C} \times S U(2)_{L} \times U(1)_{Y}$, times an additional gauge group, $U(1)_{B-L}$, whose charges are the $B-L$ quantum numbers.

In addition to having realistic spectra, the observable sector of heterotic standard models also satisfies important phenomenological constraints. Consider nucleon decay in this context. The additional $U(1)_{B-L}$ symmetry, if spontaneously broken at a low mass scale, suppresses $\Delta L=1$ and $\Delta B=1$ dimension four operators in the effective theory. These vacua exhibit natural doublet-triplet splitting [1, 21, 22]. This eliminates color triplet induced dimension five operators which can lead to rapid nucleon decay. The unification scale of het- 
erotic standard models is of $\mathcal{O}\left(10^{16} \mathrm{GeV}\right)$. Hence, nucleon decay via heavy $\operatorname{Spin}(10)$ vector bosons is sufficiently suppressed. Taken together, we see that heterotic standard models naturally reduce the nucleon decay rate to a level consistent with experimental bounds [23, 24]. Formalisms for computing the Higgs $\mu$-terms and Yukawa couplings in the observable sectors of heterotic standard models were presented in [25] and [26] respectively. In the minimal theory, for example, it was shown that the cubic moduli-Higgs-Higgs conjugate terms in the superpotential vanish due to "geometric" effects. Therefore, non-vanishing Higgs $\mu$-terms only arise from higher order interactions and, hence, are naturally suppressed [27, 28]. Minimal heterotic standard models were also shown to have an interesting texture of Yukawa couplings which renders the first quark/lepton family naturally light.

The hidden sector of heterotic standard models can be constructed in two ways. Both must have a slope-stable holomorphic vector bundle on the hidden orbifold plane. In the first approach, one allows only five-branes in the bulk space. The hidden sector bundle and the cohomology class of the five-brane are then chosen so as to saturate the anomaly cancellation condition. For heterotic standard models with five-branes, it was shown in [17] that the Chern class of the hidden sector bundle satisfies a strong necessary condition, the Bogomolov bound, for slope-stability. However, explicit slope-stable hidden sector bundles were not constructed in [4, 17]. Note that vacua of this type are $N=1$ supersymmetric. The only obvious way to break supersymmetry in this context is via gaugino condensation in the hidden sector. However, as was shown in a simplified theory in [29] and reviewed and extended to a larger context in this paper, gaugino condensation alone is not sufficient to break supersymmetry in strongly coupled heterotic vacua. Furthermore, the minimum of the moduli potential energy will have a large, negative cosmological constant, consistent with the preservation of supersymmetry. Given the fact that supersymmetry is broken at energies below the electroweak scale, and the observed very small, positive cosmological constant [30], hidden sectors of this type would not appear to be of phenomenological interest.

The second approach, which in many ways is mathematically simpler, is to allow both five-branes and anti-five-branes in the bulk space. The hidden sector bundle and the fivebrane/anti-five-brane curves are then chosen to satisfy the anomaly cancellation condition. The simplest way to do this is to take the hidden sector vector bundle to be trivial, which is trivially slope-stable. If one assumes that there are no Wilson lines in the hidden sector then the hidden sector gauge group is $E_{8}$. Given a specific Calabi-Yau threefold and observable sector bundle, the classes of the five-brane/anti-five-brane curves are explicitly fixed by the anomaly condition. The appearance of an anti-five-brane in the bulk space can potentially 
solve both problems inherent in the first approach. First of all, it explicitly breaks supersymmetry and, hence, one expects supersymmetry breaking operators in the effective theory. Secondly, as with the anti D-branes of the Type IIB theories discussed in [31, 32], anti-fivebranes give a positive contribution to the effective theory. This allows the minimum of the moduli potential energy function to be "uplifted" to a small, positive cosmological constant. Hence, the second approach to the hidden sector in heterotic standard models would appear to be more suitable to construct realistic models of particle physics and cosmology.

In this paper we will discuss the structure of heterotic standard models where the hidden sector has a trivial bundle with no Wilson lines and there are both five-branes and anti-fivebranes in the bulk space. Specifically, we will do the following. In Section 2 , we review the structure of heterotic $M$-theory vacua and present the most general Kähler potentials and superpotentials in this context. In Section 3 it is shown that in strongly coupled heterotic vacua with only five-branes in the bulk space and gaugino condensation in the hidden sector, the values of all moduli can be fixed. However, the minimum of the potential energy has a large, negative cosmological constant and does not break supersymmetry. This is proven within a slightly simplified context. Specifically, we consider the dilaton, all Kähler and complex structure moduli and the translation modulus of the bulk five-brane. However, to make the analysis tractable only a single vector bundle modulus is assumed. We also introduce string instantons on representative curves only. A further analysis of string instantons is, at present, impossible since the complete instanton series is unknown. Within this context,in Section 4, we show that the addition of an anti-five-brane to the bulk space and choosing the hidden sector bundle to be trivial continues to admit a vacuum which stabilizes all moduli. However, by appropriately choosing the Kähler moduli one can make the minimum of the potential energy have a small, positive cosmological constant consistent with the observed value. This minimum will also break $N=1$ supersymmetry. Finally, in Section 5 we apply this formalism to the moduli of the minimal heterotic standard model [4]. All moduli are stabilized in this context. Furthermore, the minimum of the potential can have a phenomenologically acceptable positive cosmological constant and break supersymmetry. This occurs for Kähler moduli for which the observable sector vector bundle is slope-stable.

Finally, we want to point out that some of the results derived in this paper were anticipated, in a simpler context, in [33, 34]. 


\section{$2 \quad \mathrm{E}_{8} \times \mathrm{E}_{8}$ Heterotic Vacua with Five-Branes}

\section{Basic Structure of the Vacuum}

In this section, we will consider $E_{8} \times E_{8}$ strongly coupled heterotic string theory compactified on the space

$$
M=\mathbb{R}^{4} \times X \times S^{1} / Z_{2},
$$

where $X$ is a Calabi-Yau threefold and $S^{1} / Z_{2}$ is an interval in the eleventh-dimension. Furthermore, we will choose the Calabi-Yau threefold $X$ to be elliptically- or torus-fibered over either an Enriques surface $\mathcal{E}$, a del Pezzo surface $d \mathbb{P}_{i}, i=1, \ldots, 9$, or a Hirzebruch surface $\mathbb{F}_{r}$, for integers $r \geq 0$. The threefold may have either trivial or non-trivial fundamental group.

Denote by $v_{C Y}$ and $\pi \rho$ the reference volume of the Calabi-Yau threefold and the reference length of the interval in the eleventh dimension respectively. The physical volume and length are obtained by multiplying them by the appropriate moduli. To achieve the correct phenomenological values for the four-dimensional Newton and gauge coupling parameters,

$$
M_{P l} \sim 10^{19} \mathrm{GeV}, \quad \alpha_{G U T} \sim \frac{1}{25},
$$

we choose the inverse reference radius of the Calabi-Yau threefold and the inverse reference length of the eleventh dimension to be

$$
v_{C Y}^{-1 / 6} \sim 10^{16} \mathrm{GeV}, \quad(\pi \rho)^{-1} \sim 10^{14} \mathrm{GeV}
$$

respectively.

Let us list the complex moduli fields arising from such a compactification. They are the dilaton $S$, the $h^{1,1}$ moduli $T^{I}$ and the $h^{2,1}$ moduli $Z_{\alpha}$. These moduli will be taken to be dimensionless. Note that

$$
\operatorname{Re} S=V, \quad \operatorname{Re} T^{I}=R a^{I} V^{-1 / 3}
$$

where $V$ is the volume modulus of the Calabi-Yau threefold, $\mathrm{R}$ is the modulus associated with the length of the $S^{1} / Z_{2}$ interval and the $a^{I}$ satisfy the constraint

$$
V=\frac{1}{6} d_{I J K} a^{I} a^{J} a^{K}
$$

with $d_{I J K}$ the Calabi-Yau intersection numbers. Given the reference lengths chosen in (2.3), the dimensionless moduli $\operatorname{Re} S$ and $R$ must be stabilized at the values

$$
\operatorname{Re} S \sim 1, \quad R \sim 1
$$


In addition to the geometrical compactification manifold (2.1), one must specify a slopestable holomorphic vector bundle on the orbifold plane at each end of the interval. On the observable brane, one chooses a vector bundle $V$ with structure group $G \subseteq E_{8}$ such that the low energy theory is supersymmetric and realistic. For example, bundles leading to the exact MSSM spectrum [4, 7], extensions of the MSSM [1, 2, 3, 35, 36, 37, 38] and GUT theories [9, 39] at low energy have been constructed. These vector bundles give rise to complex vector bundle moduli $\phi_{a}$. The hidden orbifold plane must also support a slopestable holomorphic vector bundle $V^{\prime}$ with structure group $G^{\prime} \subseteq E_{8}$. In this paper, we will always assume that $V^{\prime}$ is the trivial bundle. This bundle is trivially slope-stable and leads to a low energy hidden sector gauge group of $E_{8}$ with no additional moduli.

Finally, we allow for the possibility that there are five-branes and anti-five-branes in the bulk space between the orbifold planes. Let $[\mathcal{W}]$ and $[\overline{\mathcal{W}}]$ be the Poincare dual of the curves on which these branes and anti-branes respectively are wrapped. Then the cancellation of quantum anomalies requires that

$$
c_{2}(V)-c_{2}(T X)+[\mathcal{W}]-[\overline{\mathcal{W}}]=0
$$

Note that $c_{2}\left(V^{\prime}\right)=0$ since $V^{\prime}$ has been chosen to be trivial. Generically, the curve class satisfying (2.7) is neither effective nor anti-effective, corresponding to both wrapped fivebranes and anti-five-branes. However, in this section, we will assume that $T X$ and $V$ are such that the curve is strictly effective, leading to wrapped five-branes only. Later in the paper we will loosen this assumption and allow for anti-branes as well. We will further assume that the curve is chosen so as to correspond to the wrapping of a single five-brane. In this case, the five-brane contributes another complex modulus $\mathbf{Y}$ to the low energy theory [40, 41, 42], where

$$
\operatorname{Re} \mathbf{Y}=\frac{Y \operatorname{Re} \mathcal{T}}{\pi \rho}
$$

and $\mathrm{Y}$ is the position of the five-brane in the eleventh dimension. The superfield $\mathcal{T}$ is defined as follows. Let $\omega_{I}, I=1, \ldots, h^{1,1}$ be a basis for $H^{1,1}$ and $z^{J}, J=1, \ldots, h^{1,1}$ be the dual basis of $H_{2}$ where

$$
\frac{1}{v_{C Y}^{1 / 3}} \int_{z^{J}} \omega_{I}=\delta_{J}^{I} .
$$

The curve $z_{5}$ on which the five-brane is wrapped can be expanded as $z_{5}=c_{I} z^{I}$. Then $\mathcal{T}$ is defined to be

$$
\mathcal{T}=c_{I} T^{I}
$$


It is straightforward to show that

$$
\operatorname{Re} \mathcal{T}=\frac{1}{v_{C Y}^{1 / 3}} \int_{z_{5}} \omega_{T}
$$

where

$$
\omega_{T}=\operatorname{Re} T^{I} \omega_{I}
$$

\section{Kähler Potentials}

The Kähler potential for the $S$ and $T^{I}$ moduli was computed in [43] and is given by

$$
K_{S, T}=-M_{P l}^{2} \ln (S+\bar{S})-M_{P l}^{2} \ln \left(\frac{1}{6} d_{I J K}(T+\bar{T})^{I}(T+\bar{T})^{J}(T+\bar{T})^{K}\right) .
$$

The Kähler potential for the complex structure moduli $Z_{\alpha}$ was found in 44 to be

$$
K_{Z}=-M_{P l}^{2} \ln \left(-i \int_{X} \Omega \wedge \bar{\Omega}\right),
$$

where $\Omega$ is the holomorphic $(3,0)$ form. In [45], the Kähler potential for the five-brane modulus $\mathbf{Y}$ was calculated. It was shown that

$$
K_{5}=2 M_{P l}^{2} \tau_{5} \frac{(\mathbf{Y}+\overline{\mathbf{Y}})^{2}}{(S+\bar{S})(\mathcal{T}+\overline{\mathcal{T}})},
$$

with $\tau_{5}$ and $T_{5}$ given by

$$
\begin{aligned}
\tau_{5} & =\frac{T_{5} v_{C Y}^{1 / 3}(\pi \rho)^{2}}{M_{P l}^{2}}, \\
T_{5} & =(2 \pi)^{1 / 3} \frac{1}{\left(2 \kappa_{11}^{2}\right)^{2 / 3}} .
\end{aligned}
$$

The eleven-dimensional gravitational coupling parameter $\kappa_{11}$ is related to the four-dimensional Planck mass as

$$
\kappa_{11}^{2}=\frac{\pi \rho v_{C Y}}{M_{P l}^{2}} .
$$

Substituting (2.18) into (2.17), we can write $\tau_{5}$ as

$$
\tau_{5}=\left(\frac{\pi}{2}\right)^{1 / 3} v_{C Y}^{1 / 3}\left(\frac{(\pi \rho)^{2}}{v_{C Y} M_{P l}}\right)^{2 / 3} .
$$

For the reference parameters chosen in (2.2) and (2.3),$\tau_{5}$ becomes

$$
\tau_{5} \sim 1
$$


The Kähler potential for the vector bundle moduli is less well-known. It was shown in [33] that it always has the form

$$
\tilde{K}_{\text {bundle }}=k M_{P l}^{2} K_{\text {bundle }}\left(\phi_{a}, \bar{\phi}_{a}\right)
$$

where $k$ is a dimensionless constant given by

$$
k=\frac{1}{(4 \pi)^{5 / 3}\left((\pi \rho)^{2} M_{P l}^{2}\right)^{1 / 3}}
$$

and $K_{\text {bundle }}$ is a dimensionless function of the vector bundle moduli $\phi_{a}$. For the reference parameters given in (2.2) and (2.3),$k$ takes the value

$$
k \sim 10^{-5}
$$

The generic properties of $K_{\text {bundle }}$ relevant to moduli stabilization were discussed in detail in [46].

We conclude that

$$
K=K_{S, T}+K_{Z}+K_{5}+\tilde{K}_{\text {bundle }}
$$

is the total Kähler potential for all the moduli.

\section{Superpotentials}

There are three non-vanishing contributions to the superpotential for the moduli. First consider the flux-induced superpotential. Let us turn on a non-zero flux of the Neveu-Schwarz three-form $H$ on the Calabi-Yau threefold. The presence of this non-zero flux generates a superpotential for the $h^{2,1}$ moduli of the form [47, 48]

$$
W_{f}=M_{P l}^{3} h_{1} \int_{X} \tilde{H} \wedge \tilde{\Omega},
$$

where

$$
h_{1}=\frac{1}{v_{C Y}^{1 / 2} M_{P l}^{3}}
$$

and $\tilde{H}$ and $\tilde{\Omega}$ are both dimensionless. They have been obtain from $H$ and $\Omega$ by scaling with respect to the appropriate reference parameters. For the values chosen in (2.2) and (2.3), $h_{1}$ becomes

$$
h_{1} \sim 10^{-8}
$$


As discussed in 33], the warping away from a Calabi-Yau threefold due to the flux will be negligibly small if we take

$$
\int_{C} \tilde{H} \ll 10^{5}
$$

where $C$ is an appropriate three-cycle. Henceforth, we will always choose the flux to satisfy this condition.

Second, let us turn on a gaugino condensate on the hidden brane [29, 45, 49, 50, 51, 52, 53, 54, 55]. As discussed in [33], this produces a superpotential for the $S, T^{I}$ and Y moduli given by

$$
W_{g}=M_{P l}^{3} h_{2} \exp \left[-\epsilon\left(S-\alpha_{I}^{(2)} T^{I}+\tau_{5} \frac{\mathbf{Y}^{2}}{\mathcal{T}}\right)\right]
$$

where

$$
h_{2} \sim \frac{1}{M_{P l} v_{C Y}^{1 / 2}(\pi \rho)}\left(\frac{\kappa_{11}}{4 \pi}\right)^{2 / 3}
$$

For the values of the reference parameters chosen in (2.2) and (2.3),

$$
h_{2} \sim 10^{-6}
$$

The coefficient $\epsilon$ is related to the coefficient $b_{0}$ of the beta-function and is given by

$$
\epsilon=\frac{6 \pi}{b_{0} \alpha_{G U T}} .
$$

For the $E_{8}$ gauge group of our hidden sector $b_{0}=90$. Taking $\alpha_{G U T}$ to have its phenomenological value given in (2.2), we obtain

$$
\epsilon \sim 5
$$

The term $\alpha_{I}^{(2)} T^{I}$ is related to the tension of the hidden brane [43]. Choosing the hidden sector vector bundle to be trivial, we find that

$$
\alpha_{I}^{(2)}=-\frac{\pi \rho}{32 \pi v_{C Y}}\left(\frac{\kappa_{11}}{4 \pi}\right)^{2 / 3} \int_{z_{R}} \omega_{I},
$$

where $z_{R}$ is the curve Poincare dual to $\operatorname{Tr} R \wedge R$. One can estimate the order of magnitude of $\alpha_{I}^{(2)}$ by using the reference parameters (2.2) and (2.3) in (2.34). The result is that

$$
\alpha_{I}^{(2)} \sim \frac{1}{v_{C Y}^{1 / 3}} \int_{z_{R}} \omega_{I}
$$

The term $\tau_{5}$ was presented in (2.16). Recall, using (2.2) and (2.3), that

$$
\tau_{5} \sim 1
$$


The real part of

$$
S-\alpha_{I}^{(2)} T^{I}+\tau_{5} \frac{\mathbf{Y}^{2}}{\mathcal{T}}
$$

represents the inverse square of the gauge coupling parameter on the hidden brane, with the last two terms being threshold corrections [29, 45].

The third contribution to the moduli superpotential arises from worldsheet instantons, that is, strings wrapped on holomorphic curves in the Calabi-Yau threefold. In our context, these are generated by membranes stretching between branes. At long wavelength, such configurations reduce to strings wrapping holomorphic curves. There are three different types of membrane configurations that contribute to the superpotential.

1. A membrane stretching between the two orbifold planes.

2. A membrane beginning on the observable sector plane and ending on the five-brane.

3. A membrane beginning on the five-brane and ending on the hidden sector plane.

Let us begin with the first configuration, a membrane stretching between the two orbifold planes and wrapped on an isolated holomorphic curve $\mathcal{C}$. It was shown in [56] that its non-perturbative contribution to the superpotential has the structure

$$
W_{n p}[\mathcal{C}] \sim \operatorname{Pfaff}\left(\left.\mathcal{D}_{-}\right|_{\mathcal{C}}\right) \exp \left(-\tau \tilde{\omega}_{I} T^{I}\right)
$$

where

$$
\tau=\frac{T_{M} \pi \rho}{2} v_{C Y}^{1 / 3}
$$

and

$$
\tilde{\omega}_{I}=\frac{1}{v_{C Y}^{1 / 3}} \int_{\mathcal{C}} \omega_{I}
$$

Note that

$$
T_{M} \pi \rho=\frac{1}{2 \pi \alpha^{\prime}} .
$$

For the reference parameters in (2.2) and (2.3), $\tau$ becomes

$$
\tau \sim 10^{2} .
$$

Henceforth, $\tau$ will be assumed to be much greater than unity, which is naturally the case. The factor

$$
\operatorname{Pfaff}\left(\mathcal{D}_{-} \mid \mathcal{C}\right)
$$


in (2.38) is the Pfaffian of the chiral Dirac operator twisted by the observable sector bundle pulled back to the curve $\mathcal{C}$, see [56]. This factor has been explicitly calculated in a number of contexts [57, 58, 59] and found to be a homogeneous polynomial of the "transition" moduli of the curve $\mathcal{C}$. Note that there can be many isolated curves $\mathcal{C}$ in the Calabi-Yau threefold. It has been demonstrated that, in some contexts, the sum of the superpotential contributions from these curves vanishes identically [60]. However, in the context of this paper this is not generically the case, as shown explicitly in [58].

Now consider the second configuration, that is, a membrane stretching between the observable sector plane and the five-brane. In this case the membrane wraps on the same curve as the five-brane, namely $z_{5}$. The contribution to the superpotential is very similar to (2.38). One finds that

$$
W_{5}^{(1)} \sim \operatorname{Pfaff}\left(\left.\mathcal{D}_{-}\right|_{z_{5}}\right) e^{-\tau \mathbf{Y}}
$$

where $\tau$ is given in (2.39) and $\left.\operatorname{Pfaff}(\mathcal{D})_{-}\right|_{z_{5}}$ is (2.43) with $\mathcal{C}=z_{5}$. Similarly, the third configuration, that is, a membrane stretching from the five-brane to the hidden sector plane, contributes

$$
W_{5}^{(2)} \sim e^{-\tau(\mathcal{T}-\mathbf{Y})}
$$

to the superpotential. Note that since we choose a trivial vector bundle on the hidden sector orbifold, the Pfaffian is just unity.

\section{Moduli Stabilization}

In a typical heterotic vacuum, the number of moduli is rather large. For example, in the minimal heterotic standard model [4], there is the dilaton $S, 3=h^{1,1}$ moduli $T^{I}, 3=h^{2,1}$ moduli $Z_{\alpha}$, 13 vector bundle moduli $\phi_{a}$ and the five-brane translation modulus $\mathbf{Y}$. Other heterotic vacua often have far more moduli, especially vector bundle moduli whose number can be of $\mathcal{O}\left(10^{2}\right)$. Therefore, to obtain an explicit analytic solution for the moduli potential and its minima we must simplify the model while retaining its essential properties.

\section{$h^{1,1}=1$ Case}

It was argued in [33] that one can consider only one $h^{1,1}$ modulus and one vector bundle modulus, $T$ and $\phi$ respectively, without any loss of generality. One need not restrict the number of $h^{2,1}$ moduli $Z_{\alpha}$. Therefore, we will, in this subsection, assume that the spectrum of our vacuum consists of the moduli $S, T, Z_{\alpha}, \phi$ and $\mathbf{Y}$. 
The Kähler potential of our simplified model is given by

$$
K=K_{S, T}+K_{Z}+K_{5}+\tilde{K}_{\text {bundle }},
$$

where (2.13) becomes

$$
K_{S, T}=-M_{P l}^{2} \ln (S+\bar{S})-3 M_{P l}^{2} \ln (T+\bar{T}),
$$

expression (2.14) remains

$$
K_{Z}=-M_{P l}^{2} \ln \left(-i \int_{X} \Omega \wedge \bar{\Omega}\right)
$$

and (2.15) simplifies to

$$
K_{5}=2 M_{P l}^{2} \tau_{5} \frac{(\mathbf{Y}+\overline{\mathbf{Y}})^{2}}{(S+\bar{S})(T+\bar{T})} .
$$

In (3.2), we have chosen the Calabi-Yau intersection number to be

$$
d_{111}=1
$$

for simplicity. Any other choice of $d_{111}$ will give identical equations and leave the conclusions unchanged. Note from (2.4) and (2.5) that

$$
\operatorname{Re} T=R
$$

Also, by definition

$$
0 \leq \operatorname{Re} \mathbf{Y} \leq \operatorname{Re} T,
$$

since the five-brane must be between the orbifold planes. In the following we will always assume that

$$
|\operatorname{Im} T| \ll 1,
$$

which is required to ignore cross coupling between $T$ and $\phi$. For $\tilde{K}_{\text {bundle }}$ we choose

$$
\tilde{K}_{\text {bundle }}=k M_{P l}^{2} K_{\text {bundle }}, \quad k \sim 10^{-5},
$$

where, when $\phi$ is less than unity,

$$
K_{\text {bundle }}=-p \ln (\phi+\bar{\phi})
$$

and $p$ is a dimensionless, positive constant. Expression (3.10) is the simplest function satisfying all the requirements specified in [33]. However, one can choose $K_{\text {bundle }}$ to be any other function satisfying these requirements without altering the conclusions of this paper. 
Now consider the superpotential in our simplified vacuum. It is given by

$$
W=W_{f}+W_{g}+W_{n p}+W_{5}^{(1)}+W_{5}^{(2)},
$$

where (2.25) remains

$$
W_{f}=M_{P l}^{3} h_{1} \int_{X} \tilde{H} \wedge \tilde{\Omega}, \quad h_{1} \sim 10^{-8}
$$

and (2.29) reduces to

$$
W_{g}=M_{P l}^{3} h_{2} \exp \left[-\epsilon\left(S-\alpha^{(2)} T+\tau_{5} \frac{\mathbf{Y}^{2}}{T}\right)\right]
$$

with

$$
h_{2} \sim 10^{-6}, \quad \epsilon \sim 5, \quad \alpha^{(2)} \sim 1, \quad \tau_{5} \sim 1 .
$$

Since $h^{1,1}=1$, the space $H_{2}(X)$ is spanned by a single curve class $z$. Any other effective class is simply a positive integer multiple of $z$. For simplicity, we will take $\mathcal{C}=z_{5}=z$ in the following. Other choices for $\mathcal{C}$ and $z_{5}$ will not alter the conclusions of this subsection. It follows that the non-perturbative superpotential (2.38) is now given by

$$
W_{n p}=c_{1} M_{P l}^{3} \phi^{d+1} e^{-\tau T},
$$

where

$$
\tau \sim 10^{2},
$$

we have restored its natural scale and $c_{1}$ is some dimensionless coefficient of order unity. The Pfaffian, which must be a homogeneous polynomial, is represented by the factor $\phi^{d+1}$. We will assume that $d+1$ is sufficiently large. This is the case in explicit examples [58]. Finally, expressions (2.44) and (2.45) for the five-brane superpotentials become

$$
W_{5}^{(1)}=c_{2} M_{P l}^{3} \phi^{d+1} e^{-\tau \mathbf{Y}}
$$

and

$$
W_{5}^{(2)}=c_{3} M_{P l}^{3} e^{-\tau(T-\mathbf{Y})}
$$

respectively, where $c_{2}$ and $c_{3}$ are dimensionless coefficients of order unity.

Having specified the complete Kähler and superpotentials, one can now solve for the minimum of the moduli potential energy. Specifically, we will show that the system of equations

$$
D_{F} W=0,
$$


where $D W$ is the Kähler covariant derivative, has a solution in the correct phenomenological range for each field $F=S, T, Z_{\alpha}, \phi, \mathbf{Y}$.

To begin with, consider the equations

$$
D_{Z_{\alpha}} W=0
$$

Under the assumption that

$$
\left|W_{f}\right| \gg\left|W_{g}\right|,\left|W_{n p}\right|,\left|W_{5}^{(1)}\right|,\left|W_{5}^{(2)}\right|
$$

it was argued in [33] that (3.20) should have non-trivial solutions that fix each modulus $Z_{\alpha}$. The argument employed here is identical to that given in [31] to obtain moduli stabilization in the Type IIB context.

The remaining equations, namely

$$
D_{S} W=0, \quad D_{T} W=0, \quad D_{\mathbf{Y}} W=0, \quad D_{\phi} W=0
$$

were solved in detail in [33]. Here we simply state the results. Writing

$$
S=S_{1}+i S_{2}, \quad T=T_{1}+i T_{2} \quad \mathbf{Y}=\mathbf{Y}_{1}+i \mathbf{Y}_{2}, \quad \phi=r e^{i \theta}
$$

as well as

$$
W_{f}=\left|W_{f}\right| e^{i f},
$$

the solution to equations (3.22) were found to be the following. First,

$$
S_{1} \sim 1, \quad S_{2} \sim-\frac{f+2 \pi n_{1}}{\epsilon},
$$

where $n_{1}$ is an arbitrary integer. Note that $\operatorname{Re} S \sim 1$, as required by (2.6). Second, one finds

$$
T_{1} \sim 1, \quad T_{2} \sim-\frac{f+\pi\left(2 n_{3}+1\right)}{\tau},
$$

with $n_{3}$ an arbitrary integer. Again, $\operatorname{Re} T=R \sim 1$, as required by (2.6). Since naturally $\tau \gg 1$, one can choose $n_{3}$ so that $|\operatorname{Im} T| \ll 1$ which is consistent with assumption (3.8). Third,

$$
\mathbf{Y}_{1}=\frac{S_{1}}{2 \tau_{5}}, \quad \mathbf{Y}_{2} \sim 0
$$

Recalling that $\tau_{5} \sim 1$, we see that $\operatorname{Re} \mathbf{Y} \leq \operatorname{Re} T$, consistent with (3.7). Finally, one finds that

$$
r=\left(\frac{p k\left|W_{f}\right| e^{\tau \mathbf{Y}_{1}}}{2(d+1) c_{2} \cos (\theta)}\right)^{1 / d}, \quad \theta=\frac{f+2 \pi n_{2}}{d}
$$


Note that the five contributions to the superpotential, when evaluated at these field values, satisfy the assumption (3.21), as they must.

It is straightforward to find the value of the potential energy at this minimum. It is given by

$$
V_{\text {min }}=-3 e^{K / M_{P l}^{2}} \frac{|W|^{2}}{M_{P l}^{2}} \sim-\frac{\left|W_{f}\right|^{2}}{M_{P l}^{2}} .
$$

The size of the potential energy is, therefore, determined by the flux-induced superpotential. Since from (2.25) and (2.27) $W_{f}$ is of $\mathcal{O}\left(10^{-8} M_{P l}^{3}\right)$, we expect $V_{\min }$ to be

$$
V_{\text {min }} \sim-10^{-16} M_{P l}^{4} \sim-10^{60}(\mathrm{GeV})^{4}
$$

Note that larger values of flux can be allowed, as long as they satisfy the constraint (2.28). In this case the potential will take an even larger negative value at the minimum. Finally, since $D_{F} W=0$ in this vacuum, $N=1$ supersymmetry remains unbroken.

\section{$\mathrm{h}^{1,1}>1$ Case}

As argued in [33], there is no reason why the above analysis cannot be extended to heterotic vacua with larger numbers of Kähler and vector bundle moduli. In this section, we will generalize the above discussion to include an arbitrary number of Kähler moduli $T^{I}$, $I=$ $1, \ldots, h^{1,1}$, one vector bundle modulus $\phi$, any number of complex structure moduli $Z_{\alpha}$ and the five-brane translation modulus Y. Vacua with more than one vector bundle modulus will be considered elsewhere [61]. For specificity, we will analyze our theory in the case of $h^{1,1}=2$ Kähler moduli, $T^{1}$ and $T^{2}$. It will be clear from the discussion that this captures all relevant information and is easily generalized to arbitrary $h^{1,1}$.

The Kähler potential of our vacuum is now

$$
K=K_{S, T}+K_{Z}+K_{5}+\tilde{K}_{\text {bundle }}
$$

where $K_{Z}$ and $\tilde{K}_{\text {bundle }}$ are given by (2.14) and (3.10), (3.11) respectively. To find $K_{S, T}$ from (2.13) we must specify the intersection numbers $d_{I J K}$. Since $h^{1,1}=2$, the spaces $H^{1,1}(X)$ and $H_{2}(X)$ are spanned by $\left\{\omega_{1}, \omega_{2}\right\}$ and its dual basis $\left\{z^{1}, z^{2}\right\}$ respectively. $X$ being torus-fibered allows us to identify $z^{1}$ with the fiber class and $z^{2}$ with the curves in the base. Now, note that taking the volume of $z^{1}$ to zero must make $V$, the volume of $X$, vanish. It then follows from (2.5) that

$$
d_{222}=0
$$


Similarly, letting the volume of $z^{2}$ vanish should not send $V$ to zero. Hence, $d_{111} \neq 0$. Finally, any term involving $d_{122}$ would be sub-dominant in our analysis. Hence, we can choose the Calabi-Yau intersection numbers to vanish with the exception of

$$
d_{111}=1, \quad d_{112}=1
$$

without loss of generality. Intersection numbers of this type will appear in the minimal heterotic standard model discussed below. It follows that $K_{S, T}$ is given by (2.13) with intersection number (3.33). To specify $K_{5}$, one must specify the curve $z_{5}$ on which the fivebrane is wrapped. It is well-known [] that wrapping a string over a fiber of a torus-fibration does not contribute to the superpotential. For this reason, in this subsection we will choose

$$
z_{5}=z^{1}+z^{2} .
$$

which has a component in the base. It follows from (2.10) that

$$
\mathcal{T}=T^{1}+T^{2}
$$

$K_{5}$ is then given by (2.15) with $\mathcal{T}$ defined in (3.35). It is helpful to re-express $K_{S, T}$ in terms of fields $\mathcal{T}$ and $T^{2}$. The second term in (2.13) then becomes

$$
K_{S, T}=\cdots-M_{P l}^{2} \ln \left((\mathcal{T}+\overline{\mathcal{T}})^{3}-2(\mathcal{T}+\overline{\mathcal{T}})^{2}\left(T^{2}+\bar{T}^{2}\right)+(\mathcal{T}+\overline{\mathcal{T}})\left(T^{2}+\bar{T}^{2}\right)^{2}\right)
$$

Similarly, the superpotential is now

$$
W=W_{f}+W_{g}+W_{n p}+W_{5}^{(1)}+W_{5}^{(2)},
$$

with $W_{f}$ and $W_{g}$ given in (2.25) and (2.2.9) respectively. It is helpful to re-express the $\alpha_{I}^{(2)} T^{I}$ term in (2.29) in terms of $\mathcal{T}$ and $T^{2}$. It follows that

$$
\alpha_{I}^{(2)} T^{I}=\alpha_{1} \mathcal{T}+c T^{2}
$$

where coefficient

$$
c=\epsilon\left(\alpha_{2}^{(2)}-\alpha_{1}^{(2)}\right) .
$$

Hence, one can write $W_{g}$ as

$$
W_{g}=\left.W_{g}\right|_{\mathcal{T}} e^{c T^{2}} .
$$

To present $W_{n p}$ in (2.38), one must give the curves $\mathcal{C}$ contributing to this superpotential. In general, enumerating and specifying these curves is a very difficult problem which has not been fully solved. In this subsection, we will simplify the problem and take

$$
\mathcal{C}=z_{5}=z^{1}+z^{2}, \quad z^{2} .
$$


This choice reflects the fact that $h^{1,1}=2$ and that both $z_{5}$ and $z^{2}$ have a component in the base. Then (2.38) becomes

$$
W_{n p}=\left.W_{n p}\right|_{z_{5}}+\left.W_{n p}\right|_{z^{2}}
$$

$W_{5}^{(1)}$ and $W_{5}^{(2)}$ are given by (2.44) and (2.45) respectively. As in the $h^{1,1}=1$ case, we continue to have

$$
k \sim 10^{-5}, \quad h_{1} \sim 10^{-8}, \quad h_{2} \sim 10^{-6}, \quad \epsilon \sim 5, \quad \alpha_{1}^{(2)} \sim 1, \quad \tau_{5} \sim 1, \quad \tau \sim 10^{2} .
$$

Having specified the Kähler and superpotentials, we now solve for the minimum of the moduli potential energy. Specifically, we will show that the system of equations

$$
D_{F} W=0
$$

where $D W$ is the Kähler covariant derivative, has a solution in the correct phenomenological range for $F=S, \mathcal{T}, T^{2}, Z_{\alpha}, \phi, \mathbf{Y}$. We will denote

$$
\mathcal{T}=\mathcal{T}_{1}+i \mathcal{T}_{2}, \quad T^{2}=t_{1}+i t_{2}
$$

First consider the equations (3.44) for $F=S, \mathcal{T}, Z_{\alpha}, \phi, \mathbf{Y}$. If we assume that

$$
t_{1} \lesssim \mathcal{T}_{1}, \quad|c| t_{1} \lesssim 1, \quad\left|W_{n p}\right| z^{2}|\ll| W_{f} \mid,
$$

then these equations are, to a good approximation, the same as in the $h^{1,1}=1$ case. Hence, the solutions for $S, \mathcal{T}, Z_{\alpha}, \phi$, Y remain essential those given in (3.25), (3.26), (3.20), (3.28) and (3.27) respectively. They become identical to them for $t_{1} \ll \mathcal{T}_{1}$ and $|c| t_{1} \ll 1$. Clearly, it greatly simplifies our analysis if we can continue to use the $h^{1,1}=1$ results. For this reason, we will seek solutions under the assumption that (3.46) holds.

Now consider the $F=T^{2}$ equation

$$
\partial_{T^{2}} W=-\frac{1}{M_{P l}^{2}}\left(\partial_{T^{2}} K\right) W
$$

Using (3.28), we find that the $\left.W_{n p}\right|_{z^{2}}$ term on the left-hand side of (3.47) can be neglected relative to $W_{g}$. Then, assuming conditions (3.46) are valid, (3.47) becomes

$$
\left.W_{g}\right|_{\mathcal{T}} e^{c T^{2}} c=\frac{1}{\mathcal{T}_{1}} W_{f}
$$

In the analysis of the $D_{S} W=0$ equation, one finds that

$$
\left.W_{g}\right|_{\mathcal{T}}=-\frac{1}{2 \epsilon S_{1}} W_{f}
$$


Substituting this into (3.48) gives

$$
e^{c T^{2}}=-2 \frac{\epsilon S_{1}}{c \mathcal{T}_{1}}
$$

Writing $T^{2}=t_{1}+i t_{2}$, it follows that

$$
t_{1}=\frac{1}{c} \ln \left(\frac{2 \epsilon S_{1}}{|c| \mathcal{T}_{1}}\right)
$$

where

$$
t_{2}=\frac{\pi\left(2 n_{4}+1\right)}{c}, \quad t_{2}=\frac{2 n_{4} \pi}{c}
$$

for $c>0$ and $c<0$ respectively and $n_{4}$ is any integer. Let us parameterize

$$
c= \pm\left(\frac{2 \epsilon S_{1}}{\mathcal{T}_{1}}\right)^{1-\delta}
$$

for $c>0$ and $c<0$ respectively. Then, the solution for $t_{1}$ becomes

$$
t_{1}=|\delta|\left(\frac{2 \epsilon S_{1}}{\mathcal{T}_{1}}\right)^{\delta-1} \ln \left(\frac{2 \epsilon S_{1}}{\mathcal{T}_{1}}\right),
$$

where $\delta>0$ for $c>0$ and $\delta<0$ when $c<0$. For $|\delta| \lesssim 1, t_{1},|c| t_{1}$ and $\left|W_{n p}\right|_{z^{2}} \mid$ satisfy the assumptions in (3.46).

As an example, recall from (3.25), (3.26) and (3.43) that $S_{1} \sim 1, \mathcal{T}_{1} \sim 1$ and $\epsilon \sim 5$. These results were arrived at by implicitly choosing $\left|W_{f}\right|$ to be or order $10^{-8}$. Then

$$
c= \pm 10^{1-\delta}
$$

for $c>0$ and $c<0$ respectively and $t_{1}$ becomes

$$
t_{1}=|\delta|\left(10^{\delta-1} \ln (10)\right),
$$

where $\delta>0$ for $c>0$ and $\delta<0$ when $c<0$. Note that $\alpha_{2}^{(2)}-\alpha_{1}^{(2)} \sim .2$ when $|\delta|=1$ and increases to $\alpha_{2}^{(2)}-\alpha_{1}^{(2)}=2$ for $|\delta| \ll 1$. These are realistic values for the type of Calabi-Yau threefolds we are considering. The general situation is the following. One chooses a torusfibered Calabi-Yau threefold with $h^{1,1}=2$ and computes $\alpha_{2}^{(2)}-\alpha_{1}^{(2)}$. This is expected to be a number of $\mathcal{O}(1)$. Then adjust the flux superpotential so that

$$
1-\frac{\left|\alpha_{2}^{(2)}-\alpha_{1}^{(2)}\right|}{2 S_{1} / \mathcal{T}_{1}} \sim \mathcal{O}(\delta) .
$$

In this way one fine-tunes the left-hand expression to give $\delta$ and, hence, $t_{1}$ of the desired value. We will explore this in more generality elsewhere. 
We conclude that in the $h^{1,1}=2$ case, there exists a solution of the equations $D_{F} W=0$ for $F=S, \mathcal{T}, T^{2}, Z_{\alpha}, \phi, \mathbf{Y}$. The values for $S, \mathcal{T}, Z_{\alpha}, \phi, \mathbf{Y}$ are those given in the $h^{1,1}=1$ case and $T^{2}=t_{1}+i t_{2}$, where

$$
t_{1}=|\delta|\left(\frac{2 \epsilon S_{1}}{\mathcal{T}_{1}}\right)^{\delta-1} \ln \left(\frac{2 \epsilon S_{1}}{\mathcal{T}_{1}}\right),
$$

where $\delta>0$ for $c>0$ and $\delta<0$ when $c<0$ and

$$
t_{2}=\frac{\pi\left(2 n_{4}+1\right)}{c}, \quad t_{2}=\frac{2 n_{4} \pi}{c}
$$

for $c>0$ and $c<0$ respectively and $n_{4}$ is any integer. Note that since $t_{1} \lesssim \mathcal{T}_{1}$, it follows from (2.4), (2.5) and (3.33) that $\operatorname{Re} \mathcal{T} \sim R$. Hence, we see from (3.25) and (3.26) that $V$ and $R$ continue to be stabilized at the phenomenologically acceptable values of

$$
V \sim 1, \quad R \sim 1
$$

Since $\left|W_{f}\right|$ continues to dominate the contributions to the superpotential, we have

$$
V_{\text {min }} \sim-10^{-16} M_{P l}^{4} \sim-10^{60}(\mathrm{GeV})^{4},
$$

as in the $h^{1,1}$ case. Since $D_{F} W=0$ for all fields $F, N=1$ supersymmetry remains unbroken in this vacuum.

We considered the case $h^{1,1}=2$ case for specificity only. The analysis is easily repeated for any number of Kähler moduli $T^{I}, I=1, \ldots, h^{1,1}$ when $h^{1,1}>2$. The solutions are similar and easily found and the same conclusions will hold.

\section{Results}

The above results are based on our analysis of simplified, but representative, heterotic $M$ theory vacua with a non-trivial, slope-stable holomorphic vector bundle in the observable sector, a trivial vector bundle without Wilson lines in the hidden sector and a holomorphic five-brane in the bulk space. We found the following.

- For a natural range of parameters, the potential energy function of the moduli fields has a minimum which fixes the values of all moduli. Both $\operatorname{Re} S$ and $R$ at this minimum are of $\mathcal{O}(1)$, as required on phenomenological grounds. The vacuum value of $\operatorname{Re} \mathbf{Y}$ is between 0 and $R$, as it must be. 
- The potential energy function evaluated at this minimum is negative. Its value is typically large, of order $V_{\min } \sim-10^{-16} M_{P l}^{4}$. Hence, this theory has a large, negative cosmological constant.

- The Kähler covariant derivatives $D W$ vanish for all moduli fields at this minimum. Hence, supersymmetry is unbroken in this vacuum, despite the occurrence of gaugino condensation in the hidden sector.

Despite the fact that these results were derived in a simplified model, we see no reason that they do not apply to heterotic vacua with larger numbers of vector bundle moduli, as argued in 33]. We note that our conclusions are consistent with the results of [34], in the heterotic $M$-theory context, and 62, 63, 64, 65, 66, 67, 68, 69, 70, 71, 72], in the context of Type IIB superstrings. In the case of Type IIB strings, it was shown in 31] that one could "lift" the minimum of the moduli potential to a positive value by adding anti D-branes to the theory. The new minimum continues to fix all moduli, has a phenomenologically acceptable small positive cosmological constant, albeit by fine-tuning 73], and, due to the presence of anti D-branes, breaks supersymmetry. As was discussed in [17], a topologically stable configuration of five-branes and anti-five-branes occurs naturally in the heterotic standard models presented in 1, 2, 3, 4]. It is reasonable, therefore, to ask whether the existence of anti-five-branes in a heterotic $M$-theory vacuum might, when combined with the results of this section, lead to a vacuum with a small, positive cosmological constant that breaks supersymmetry and fixes all moduli. The answer is affirmative. This is the content of the following section.

As a final comment, note that above we have solved the $D_{F} W=0$ equations under the assumption that the fields satisfy (3.46). This allowed us to use the solutions for $S, \mathcal{T}$, $Z_{\alpha}, \phi$ and $\mathbf{Y}$ obtained in the $h^{1,1}=1$ case and, hence, to simplify the analysis. However, this assumption is not necessary. If at least one of the conditions in (3.46) is not imposed, then one would have to search for new solutions for all of the $D_{F} W=0$ equations. Since these can be continuously relaxed to the equations discussed above, we see no reason why solutions would not exist. That is, we expect there to be supersymmetric vacua with a negative cosmological constant over a wide range of moduli space. However, we have not verified this explicitly. 


\section{Adding Anti-Five-Branes}

In 31], it was argued that the vacuum state of flux-compactifications with $D$-branes and gaugino condensation in certain Type IIB theories completely fixes all moduli fields, albeit with a large, negative cosmological constant and without breaking supersymmetry. It was then shown that introducing an anti D-brane into such a vacuum adds a term proportional to the tension of the anti $D$-brane to the original $N=1$ supersymmetric Lagrangian. This additional term was shown to lift the minimum to a meta-stable vacuum that fixes all moduli, breaks the $N=1$ supersymmetry and has, after fine-tuning, a phenomenologically acceptable small, positive cosmological constant. This mechanism has recently been studied in more detail in [74, 75], who reach the same conclusion and explicitly compute the induced supersymmetry breaking terms.

In the context of heterotic $M$-theory, we have seen in the previous section that the vacuum state of flux-compactifications with five-branes and gaugino condensation fixes all moduli, but with a large, negative cosmological constant and without supersymmetry breaking. Following [31], we now add an anti-five-brane in the vacuum state. One can perform, in the heterotic context, a calculation similar to that described in [31]. This was carried out in [34] with the result that, again, the original $N=1$ supersymmetric Lagrangian is modified by the addition of a term proportional to the tension of the anti-five-brane. Specifically, this term was found to be

$$
\Delta U_{\overline{5}}=\frac{4 T_{5}}{V^{4 / 3} R^{2}} \int_{X} \omega \wedge J
$$

where

$$
J=c_{2}(V)-c_{2}(T X)+[\mathcal{W}]+[\overline{\mathcal{W}}]
$$

the integral is with respect to the Kähler form

$$
\omega=a^{I} \omega_{I}
$$

and

$$
T_{5}=(2 \pi)^{1 / 3}\left(\frac{1}{2 \kappa_{11}^{2}}\right)^{2 / 3}, \quad \kappa_{11}^{2}=\frac{\pi \rho v_{C Y}}{M_{P l}^{2}} .
$$

In (4.2), $[\mathcal{W}]$ is the cohomology class of the wrapped five-brane, $[\overline{\mathcal{W}}]$ is the class of the wrapped anti-five-brane and we have used the fact that the hidden sector bundle $V^{\prime}$ has been chosen to be trivial. The first two terms in (4.2) arise from the "end-of-the-world" orbifold planes. The third and fourth terms are generated by the five-brane and anti-fivebrane respectively. Quantum consistency demands that the theory be anomaly free. It 
follows that one must require that

$$
c_{2}(V)-c_{2}(T X)+[\mathcal{W}]-[\overline{\mathcal{W}}]=0
$$

and, hence, (4.2) becomes

$$
J=2[\overline{\mathcal{W}}] .
$$

It is important to note that if there was no anti-five-brane in the vacuum $\mathrm{J}$ and, hence, $\Delta U_{\overline{5}}$ would vanish. However, in the presence of an anti-five-brane one has a non-zero addition to the potential energy given by

$$
\Delta U_{\overline{5}}=8 T_{5} \mathcal{V}_{\overline{5}} \frac{1}{V^{4 / 3} R^{2}}
$$

where

$$
\mathcal{V}_{\overline{5}}=\int_{z_{\overline{5}}} \omega
$$

is the volume of the curve $z_{\overline{5}}$ on which the anti-five-brane is wrapped.

Given (4.7), one can add it to the $N=1$ supersymmetric Lagrangian discussed in the previous section, find the new equations of motion and re-solve for the vacuum solution. We find results completely consistent with those presented in 34] and [31] for the Type IIB string. First, the theory continues to possess a local minimum that fixes all moduli. It is related to the supersymmetric vacuum found in the previous section in the sense that it continuously relaxes to it as $\Delta U_{\overline{5}}$ is switched off. Second, the values of the moduli at this minimum are not significantly altered over those in the supersymmetric vacuum. In particular, one still finds that

$$
V \sim 1, \quad R \sim 1
$$

Third, the addition of $\Delta U_{\overline{5}}$ to the Lagrangian provides a source of positive energy, uplifting the value of the potential at the minimum. Comparing (4.7) and (4.8) with (3.30), and using (4.9), we see that the cosmological constant at the uplifted vacuum can be made positive with an arbitrarily small value as long as one chooses

$$
T_{5} \mathcal{V}_{\overline{5}} \sim 10^{-16} \mathrm{GeV}
$$

or, equivalently, using (2.2), (2.3) and (4.4) that

$$
\frac{\mathcal{V}_{\overline{5}}}{v_{C Y}^{1 / 3}} \sim 10^{-7} \text {. }
$$

Four, $N=1$ supersymmetry is broken in the uplifted vacuum. Fifth, as in the Type IIB theories studied in [31], this uplifted vacuum is now meta-stable, with a possibility to tunnel 
to large values of $\operatorname{Re} S$ and $\operatorname{Re} \mathcal{T}$. The lifetime of this meta-stable vacuum, however, will be very long. And sixth, recall that $\operatorname{Re} \mathcal{T} \sim 1$ for any $h^{1,1} \geq 1$. It then follows from (2.4), (2.11), (4.3), and (4.9) that

$$
\frac{\mathcal{V}_{5}}{v_{C Y}^{1 / 3}} \sim 1 .
$$

All of the above properties are straightforward, if tedious, to prove and are very similar to the calculations required in the Type IIB case. Hence, we won't discuss them further here. A detailed analysis of the supersymmetry breaking terms along the lines of [74, 75] will be presented elsewhere. What is necessary for us to prove here is that the conditions (4.11) and (4.12) can consistently be achieved in our context.

Let us show that this is possible for the $h^{1,1}=2$ case discussed in the previous section. Recall that $z_{5}=z^{1}+z^{2}$ is the curve on which the five-brane is wrapped. Then, its volume is given by

$$
\mathcal{V}_{5}=\int_{z_{5}=z^{1}+z^{2}} \omega
$$

Using (2.9) and (4.3) we see that

$$
\frac{\mathcal{V}_{5}}{v_{C Y}^{1 / 3}}=a^{1}+a^{2} .
$$

Now assume that there is an anti five-brane added to the theory which is wrapped on the curve $z^{2}$. Then its volume is

$$
\mathcal{V}_{\overline{5}}=\int_{z^{2}} \omega
$$

which, using (2.9) and (4.3), becomes

$$
\frac{\mathcal{V}_{\overline{5}}}{v_{C Y}^{1 / 3}}=a^{2} .
$$

In this vacuum, $t_{1}$ and, hence, $a^{2}$ are much smaller than unity. It then follows from (2.5) that $a^{1} \sim V^{1 / 3}$. Recalling that $V \sim 1$, expression (4.14) becomes

$$
\frac{\mathcal{V}_{5}}{v_{C Y}^{1 / 3}} \sim 1 .
$$

Now note from (3.58) and (4.9) that $a^{2} \sim \mathcal{O}(\delta)$. By fine-tuning $\delta$ to be small, one can set

$$
\frac{\mathcal{V}_{\overline{5}}}{v_{C Y}^{1 / 3}} \sim 10^{-7},
$$

as desired. Thus, in the $h^{1,1}=2$ vacuum discussed above, conditions (4.11) and (4.12) can be simultaneously satisfied. 
In a general vacuum, to obtain a small, positive cosmological constant, one must work in a region of Kähler moduli space in which (4.11) and (4.12) are simultaneously satisfied. Furthermore, assuming that such a region exists, it must be demonstrated that the observable sector vector bundle $V$ is slope-stable with respect to at least one such Kähler modulus. Fortunately, all of these conditions can be simultaneously satisfied. To demonstrate this requires that one give an explicit vacuum. In particular, it is necessary to present the fivebrane curve $z_{5}$, the anti-five-brane curve $z_{\overline{5}}$, a holomorphic vector bundle $V$ on the observable sector and the explicit region of the Kähler cone for which $V$ is slope-stable. We will do this in the next section for the minimal heterotic standard model.

\section{$5 \quad$ Minimal Heterotic Standard Model}

The minimal heterotic standard model was presented in 4]. The observable sector consists of a holomorphic vector bundle $V$ with structure group $S U(4)$ over a torus-fibered CalabiYau threefold $X$ with fundamental group $\mathbb{Z}_{3} \times \mathbb{Z}_{3}$. This leads to a low energy theory whose matter content in the observable sector is exactly that of the MSSM. As discussed in [17], it is easiest to choose a trivial bundle $V^{\prime}=\mathcal{O}_{X}$ in the hidden sector. The number and properties of five-branes and anti-five-branes in the bulk space are then determined by the requirement that the theory be anomaly free.

\section{Minimal Heterotic Standard Model}

The Calabi-Yau threefold $X$ is constructed from a covering space $\tilde{X}$ on which $\mathbb{Z}_{3} \times \mathbb{Z}_{3}$ acts freely. Then

$$
X=\tilde{X} /\left(\mathbb{Z}_{3} \times \mathbb{Z}_{3}\right) .
$$

Denote the quotient map as $q: \tilde{X} \rightarrow X$. It was shown in 15] that $H^{1,1}(\tilde{X})^{\mathbb{Z}_{3} \times \mathbb{Z}_{3}}$ is threedimensional and spanned by the cohomology classes $\tau_{1}, \tau_{2}$ and $\phi$. It follows that $H^{1,1}(X)$ is also three-dimensional and spanned by $\omega_{I}, I=1,2,3$ where $q^{*} \omega_{1}=\tau_{1}, q^{*} \omega_{2}=\tau_{2}$ and $q^{*} \omega_{3}=\phi$. The intersection numbers of $X$ are defined to be

$$
d_{I J K}=\frac{1}{v_{C Y}} \int_{X} \omega_{I} \wedge \omega_{J} \wedge \omega_{K}
$$

By pulling this expression back to $\tilde{X}$, one can compute these coefficients. We find that

$$
\begin{gathered}
d_{112}=d_{121}=d_{211}=\frac{1}{3}, \quad d_{122}=d_{212}=d_{221}=\frac{1}{3}, \\
d_{123}=d_{132}=d_{213}=d_{231}=d_{312}=d_{321}=1 .
\end{gathered}
$$


Note that these intersection numbers are mathematically similar to the those presented in (3.33). Specifically, they imply that $z^{1}, z^{2}$ and $z^{3}$ in the minimal heterotic standard model correspond to $z^{1}$ and $z^{2}$ in the $h^{1,1}=2$ case, respectively. There are certain codimension one boundaries in $H^{1,1}(X)$ where geometric transitions occur, that is, where the volume of at least one curve vanishes. To exclude these regions, one must choose a Kähler form in the three-dimension Kähler cone [16]

$$
\mathcal{K}=\left\{a^{1} \omega_{1}+a^{2} \omega_{2}+a^{3} \omega_{3} \mid a^{1}, a^{2}, a^{3}>0\right\} \subset H^{1,1}(X) .
$$

Henceforth, we will consider only Kähler classes

$$
\omega=a^{1} \omega_{1}+a^{2} \omega_{2}+a^{3} \omega_{3} \in \mathcal{K} .
$$

Recall from (2.5) that $V=\frac{1}{6} d_{I J K} a^{I} a^{J} a^{K}$. It follows from (5.4) that

$$
V=\frac{1}{6}\left(\left(a^{1}\right)^{2} a^{2}+a^{1}\left(a^{2}\right)^{2}+6 a^{1} a^{2} a^{3}\right) .
$$

The slope-stability of the observable sector vector bundle $V$ was proven in [17]. Specifically,

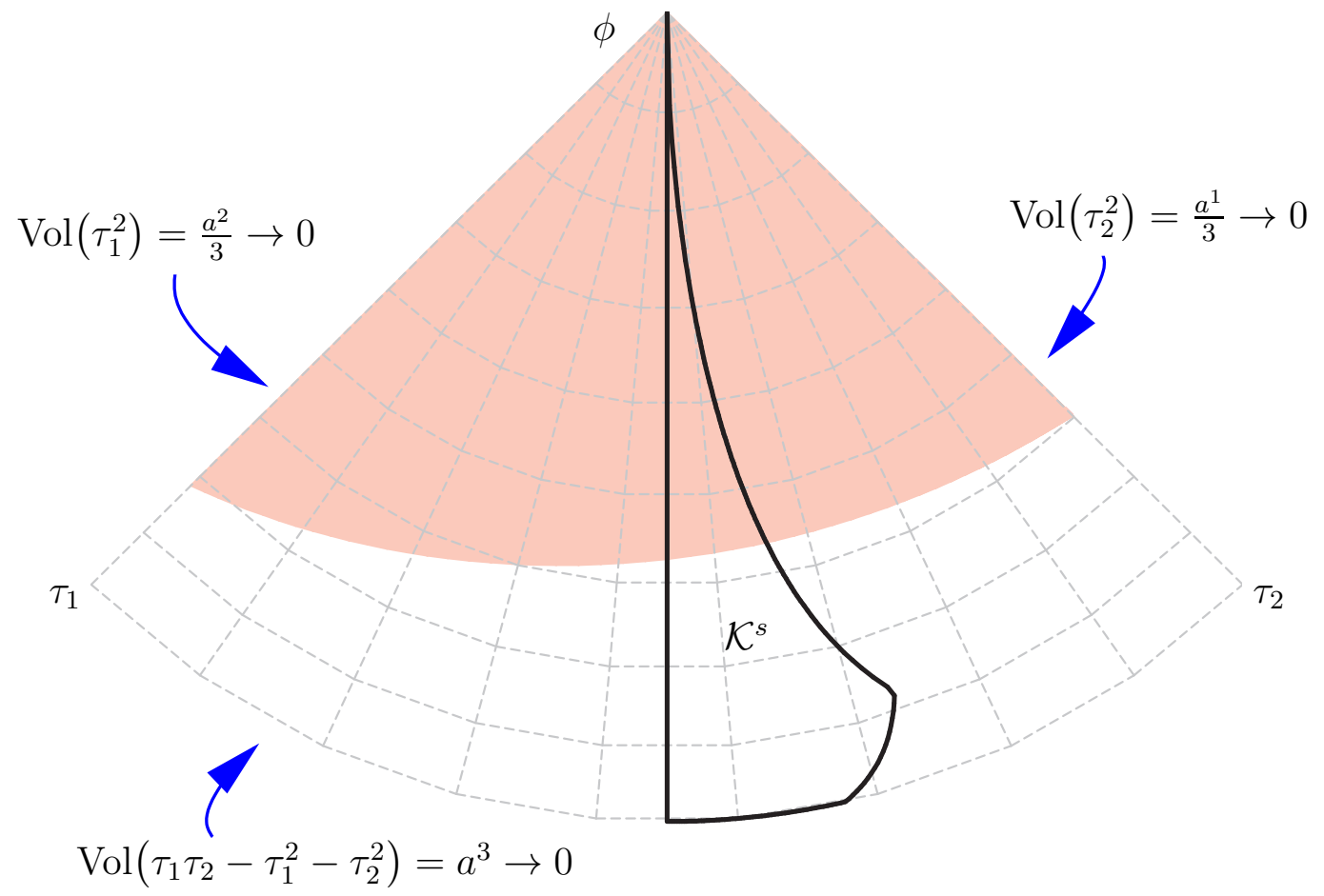

Figure 1: Kähler Cone. The observable sector vector bundle is slope-stable in the region $\mathcal{K}^{s}$.

it was shown that $V$ is slope-stable with respect to any Kähler modulus in a restricted threedimensional region $\mathcal{K}^{s}$ of the Kähler cone $\mathcal{K}$. As will be important in the following, one can

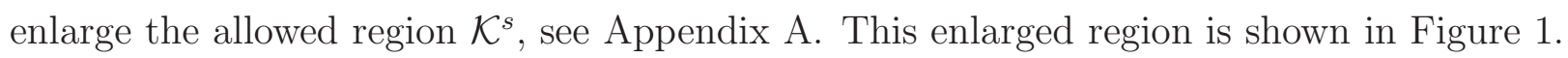




\section{Volumes of Curves}

It was shown in [4] that

$$
c_{2}(V)=\omega_{1}^{2}+4 \omega_{2}^{2}+4 \omega_{1} \omega_{2}, \quad c_{2}(T X)=12\left(\omega_{1}^{2}+\omega_{2}^{2}\right)
$$

The cancellation of quantum anomalies requires that

$$
c_{2}(V)-c_{2}(T X)+[\mathcal{W}]-[\overline{\mathcal{W}}]=0
$$

where we have used the fact that $V^{\prime}=\mathcal{O}_{X}$. Inserting (5.4), this condition becomes

$$
[\mathcal{W}]-[\overline{\mathcal{W}}]=11 \omega_{1}^{2}+8 \omega_{2}^{2}-4 \omega_{1} \omega_{2}=\left(3 \omega_{1}^{2}\right)+4\left(\omega_{1}^{2}+\omega_{2}^{2}\right)-4\left(\omega_{1} \omega_{2}-\omega_{1}^{2}-\omega_{2}^{2}\right) .
$$

Note that the terms in brackets are Poincare dual to effective curves on $X$. Since they appear with positive and negative coefficients the overall curve is not effective, and we require a nonvanishing anti-five-brane. The simplest way to cancel the anomaly is to set

$$
[\mathcal{W}]=7 \omega_{1}^{2}+4 \omega_{2}^{2} \quad[\overline{\mathcal{W}}]=4\left(\omega_{1} \omega_{2}-\omega_{1}^{2}-\omega_{2}^{2}\right)
$$

which we will do henceforth.

The volumes of the five-brane curve $z_{5}$ and the anti-five-brane curve $z_{\overline{5}}$ are easily computed. First consider the anti-five-brane curve. Note that

$$
\frac{\mathcal{V}_{\overline{5}}}{v_{C Y}^{1 / 3}}=\frac{1}{v_{C Y}^{1 / 3}} \int_{z_{\overline{5}}} \omega=\frac{1}{v_{C Y}} \int_{X} \omega \wedge[\overline{\mathcal{W}}] .
$$

It then follows from (5.3), (5.5) and (5.10) that

$$
\frac{\mathcal{V}_{\overline{5}}}{v_{C Y}^{1 / 3}}=4 a^{3} \text {. }
$$

Similarly, for the five-brane curve

$$
\frac{\mathcal{V}_{5}}{v_{C Y}^{1 / 3}}=\frac{1}{v_{C Y}^{1 / 3}} \int_{z_{5}} \omega=\frac{1}{v_{C Y}} \int_{X} \omega \wedge[\mathcal{W}]
$$

which, using (5.3), (5.5) and (5.10), becomes

$$
\frac{\mathcal{V}_{5}}{v_{C Y}^{1 / 3}}=\frac{4}{3} a^{1}+\frac{7}{3} a^{2} .
$$

Now consider the region $\mathcal{K}^{s}$ in Figure 1. Note that as one approaches the bottom of $\mathcal{K}^{s}$ the modulus $a^{3} \rightarrow 0$. It follows from (15.12) that $\frac{\mathcal{V}_{5}}{v_{C Y}^{1 / 3}}$ can be made arbitrarily small. In particular, for the appropriate value of $a^{3}$ one can set

$$
\frac{\mathcal{V}_{\overline{5}}}{v_{C Y}^{1 / 3}} \sim 10^{-7}
$$


as required by (4.11). Now note that the region $\mathcal{K}^{s}$ is bounded on the left by the vertical line defined by $a^{1}=a^{2}$. For very small values of $a^{3}$, it follows from (5.6) and the fact that $V \sim 1$ in this vacuum that $a^{1} \cong a^{2} \sim(3)^{1 / 3}$. Therefore, for moduli inside of $\mathcal{K}^{s}$ near the vertical line as $a^{3} \rightarrow 0$, one finds

$$
\frac{\mathcal{V}_{5}}{v_{C Y}^{1 / 3}} \sim 1
$$

as required by (4.12).

\section{Results}

For the minimal heterotic standard model we have shown the following.

- Taking the hidden sector vector bundle to be trivial, the anomaly cancellation condition specifies that this vacuum has both a five-brane and an anti-five-brane in the $S^{1} / \mathbb{Z}_{2}$ interval and uniquely fixes their cohomology classes.

- Neglecting the anti-five-brane, this vacuum has the structure of the $h^{1,1}>1$ theories analyzed in Section 3. As a consequence, neglecting the anti-five-brane, all moduli are stabilized, but at an $N=1$ supersymmetry preserving minimum with $V_{\min } \sim$ $-10^{-16} M_{P l}^{4}$.

- Adding the anti-five-brane lifts the minimum to a meta-stable vacuum with a positive cosmological constant. The moduli are fixed in this vacuum and have phenomenologically acceptable values.

- There is a region of the Kähler cone for which the cosmological constant has its experimental value and for which the observable sector vector bundle is slope-stable. One expects that the Kähler moduli of the meta-stable vacuum can be fine-tuned to lie in this region.

\section{Acknowledgments}

We are grateful to E. Buchbinder, R. Donagi, B. Nelson, T. Pantev, and M. Schulz for enlightening discussions. This research was supported in part by the Department of Physics and the Math/Physics Research Group at the University of Pennsylvania under cooperative research agreement DE-FG02-95ER40893 with the U. S. Department of Energy and an NSF Focused Research Grant DMS0139799 for "The Geometry of Superstrings." 


\section{A Improved Stability Bound}

Showing that the vector bundle $V$ on $X$ is stable is equivalent to showing that $\widetilde{V}$ on $\widetilde{X}$ is equivariantly stable. This can be done by finding a set of inequalities that the Kähler moduli have to satisfy [16], and then showing that a common solution exist. For the minimal heterotic standard model, this has been worked out in [17].

To find the potentially destabilizing sub-bundles one has to decide if there are maps

$$
\begin{aligned}
& \operatorname{Hom}\left(\mathcal{O}_{B_{1}}(-4 t+2 f), \mathcal{O}_{B_{1}}(-t+f) \otimes I_{3}\right)=\operatorname{Hom}\left(\mathcal{O}_{B_{1}}, \mathcal{O}_{B_{1}}(3 t-f) \otimes I_{3}\right), \\
& \operatorname{Hom}\left(\mathcal{O}_{B_{2}}(-4 t+2 f), \mathcal{O}_{B_{2}}(-t+f) \otimes I_{6}\right)=\operatorname{Hom}\left(\mathcal{O}_{B_{2}}, \mathcal{O}_{B_{2}}(3 t-f) \otimes I_{6}\right) .
\end{aligned}
$$

If there are maps in eq. A.1a , then the line bundles $\mathcal{O}_{\widetilde{X}}\left(-4 \tau_{1}+\tau_{2}+2 f\right)$ can map to $\widetilde{\mathcal{V}}$, and hence must have negative slope (or $\widetilde{\mathcal{V}}$ is rendered unstable). On the other hand, if there is no map then $\mathcal{O}_{\widetilde{X}}\left(-4 \tau_{1}+\tau_{2}+2 f\right)$ imposes no restriction on the stability of $\widetilde{\mathcal{V}}$. In the proof of slope-stability presented in [17], it was always assumed that there are maps of the form eqns. A.1a (A.1b). This leads to correct, but too strong, inequalities for the Kähler moduli.

In fact, there are no maps in eqns. A.1a , A.1b. Recall that there is a single section of $\mathcal{O}_{B_{i}}(3 t-f)$,

$$
H^{0}\left(\tilde{X}, \mathcal{O}_{B_{i}}(3 t-f)\right)=\mathbb{C}
$$

unique up to scale. Its zero locus are 9 disjoint sections $\mathbb{P}^{1} \subset B_{i}$, permuted by the $\mathbb{Z}_{3} \times \mathbb{Z}_{3}$ action. Furthermore, the points in the ideal sheaves $I_{3}, I_{6}$ are the singular points of $I_{1}$ Kodaira fibers, which do not meet any smooth sections. Therefore,

$$
\operatorname{Hom}\left(\mathcal{O}_{B_{1}}, \mathcal{O}_{B_{1}}(3 t-f) \otimes I_{3}\right)=\operatorname{Hom}\left(\mathcal{O}_{B_{2}}, \mathcal{O}_{B_{2}}(3 t-f) \otimes I_{6}\right)=0
$$

Using this result, the Proposition 1 in [17] can be strengthened to

Proposition 1 If all line bundles $\mathcal{O}_{\widetilde{X}}\left(a_{1} \tau_{1}+a_{2} \tau_{2}+b \phi\right)$ with

$$
\begin{aligned}
\left(a_{1}, a_{2}, b\right) \in\{(1,-1,-1),(-1,1,-1),(-2,2,0),(2,-2,-1), & (2,-5,1),(1,-4,1),(-4,1,1)\}
\end{aligned}
$$

have negative slope then the vector bundle $\widetilde{\mathcal{V}}$ is equivariantly stable.

The set $\mathcal{K}^{s}$ of Kähler moduli such that all slopes are indeed negative is shown in Figure 1. 


\section{Bibliography}

[1] V. Braun, Y.-H. He, B. A. Ovrut, and T. Pantev, A heterotic standard model, Phys. Lett. B618 (2005) 252-258, hep-th/0501070.

[2] V. Braun, Y.-H. He, B. A. Ovrut, and T. Pantev, A standard model from the e(8) $x$ e(8) heterotic superstring, JHEP 06 (2005) 039, hep-th/0502155.

[3] V. Braun, Y.-H. He, B. A. Ovrut, and T. Pantev, Vector bundle extensions, sheaf cohomology, and the heterotic standard model, hep-th/0505041.

[4] V. Braun, Y.-H. He, B. A. Ovrut, and T. Pantev, The exact mssm spectrum from string theory, hep-th/0512177.

[5] B. A. Ovrut, A heterotic standard model, AIP Conf. Proc. 805 (2006) 236-239.

[6] W. Buchmuller, K. Hamaguchi, O. Lebedev, and M. Ratz, The supersymmetric standard model from the heterotic string, hep-ph/0511035.

[7] V. Bouchard and R. Donagi, An su(5) heterotic standard model, Phys. Lett. B633 (2006) 783-791, hep-th/0512149.

[8] V. Bouchard, M. Cvetic, and R. Donagi, Tri-linear couplings in an heterotic minimal supersymmetric standard model, hep-th/0602096.

[9] B. R. Greene, K. H. Kirklin, P. J. Miron, and G. G. Ross, A three generation superstring model. 1. compactification and discrete symmetries, Nucl. Phys. B278 (1986) 667.

[10] B. R. Greene, K. H. Kirklin, P. J. Miron, and G. G. Ross, A three generation superstring model. 2. symmetry breaking and the low-energy theory, Nucl. Phys. B292 (1987) 606.

[11] B. R. Greene, K. H. Kirklin, P. J. Miron, and G. G. Ross, A superstring inspired standard model, Phys. Lett. B180 (1986) 69.

[12] R. Blumenhagen, G. Honecker, and T. Weigand, Non-abelian brane worlds: The open string story, hep-th/0510050.

[13] R. Blumenhagen, S. Moster, and T. Weigand, Heterotic gut and standard model vacua from simply connected calabi-yau manifolds, hep-th/0603015 
[14] B. Andreas and D. Hernandez Ruiperez, $U(n)$ vector bundles on calabi-yau threefolds for string theory compactifications, hep-th/0410170.

[15] V. Braun, B. A. Ovrut, T. Pantev, and R. Reinbacher, Elliptic calabi-yau threefolds with $z$ (3) $x$ z(3) wilson lines, JHEP 12 (2004) 062, hep-th/0410055.

[16] T. L. Gomez, S. Lukic, and I. Sols, Constraining the kaehler moduli in the heterotic standard model, hep-th/0512205

[17] V. Braun, Y.-H. He, and B. A. Ovrut, Stability of the minimal heterotic standard model bundle, hep-th/0602073.

[18] P. Langacker, Neutrino physics (theory), Int. J. Mod. Phys. A20 (2005) 5254-5265, hep-ph/0411116.

[19] J. Giedt, G. L. Kane, P. Langacker, and B. D. Nelson, Massive neutrinos and (heterotic) string theory, Phys. Rev. D71 (2005) 115013, hep-th/0502032.

[20] V. Braun, Y.-H. He, B. A. Ovrut, and T. Pantev, Heterotic standard model moduli, JHEP 01 (2006) 025, hep-th/0509051.

[21] E. Witten, Deconstruction, g(2) holonomy, and doublet-triplet splitting, hep-ph/0201018.

[22] R. Donagi, Y.-H. He, B. A. Ovrut, and R. Reinbacher, Higgs doublets, split multiplets and heterotic su(3) c x su(2)l x u(1)y spectra, Phys. Lett. B618 (2005) 259-264, hep-th/0409291.

[23] P. Nath and P. F. Perez, Proton stability in grand unified theories, in strings, and in branes, hep-ph/0601023.

[24] R. Tatar and T. Watari, Proton decay, yukawa couplings and underlying gauge symmetry in string theory, hep-th/0602238.

[25] V. Braun, Y.-H. He, B. A. Ovrut, and T. Pantev, Moduli dependent mu-terms in a heterotic standard model, hep-th/0510142.

[26] V. Braun, Y.-H. He, and B. A. Ovrut, Yukawa couplings in heterotic standard models, hep-th/0601204. 
[27] G. F. Giudice and A. Masiero, A natural solution to the mu problem in supergravity theories, Phys. Lett. B206 (1988) 480-484.

[28] S. Weinberg, The quantum theory of fields. vol. 3: Supersymmetry, . Cambridge, UK: Univ. Pr. (2000) 419 p.

[29] A. Lukas, B. A. Ovrut, and D. Waldram, Gaugino condensation in m-theory on s**1/z(2), Phys. Rev. D57 (1998) 7529-7538, hep-th/9711197.

[30] Supernova Search Team Collaboration, A. G. Riess et. al., Observational evidence from supernovae for an accelerating universe and a cosmological constant, Astron. J. 116 (1998) 1009-1038, astro-ph/9805201.

[31] S. Kachru, R. Kallosh, A. Linde, and S. P. Trivedi, De sitter vacua in string theory, Phys. Rev. D68 (2003) 046005, hep-th/0301240.

[32] S. Kachru, J. Pearson, and H. L. Verlinde, Brane/flux annihilation and the string dual of a non- supersymmetric field theory, JHEP 06 (2002) 021, hep-th/0112197.

[33] E. I. Buchbinder and B. A. Ovrut, Vacuum stability in heterotic m-theory, Phys. Rev. D69 (2004) 086010, hep-th/0310112.

[34] E. I. Buchbinder, Raising anti de sitter vacua to de sitter vacua in heterotic m-theory, Phys. Rev. D70 (2004) 066008, hep-th/0406101.

[35] R. Donagi, B. A. Ovrut, T. Pantev, and D. Waldram, Standard-model bundles on non-simply connected calabi-yau threefolds, JHEP 08 (2001) 053, hep-th/0008008.

[36] R. Donagi, Y.-H. He, B. A. Ovrut, and R. Reinbacher, The particle spectrum of heterotic compactifications, JHEP 12 (2004) 054, hep-th/0405014.

[37] R. Donagi, Y.-H. He, B. A. Ovrut, and R. Reinbacher, Moduli dependent spectra of heterotic compactifications, Phys. Lett. B598 (2004) 279-284, hep-th/0403291.

[38] R. Donagi, Y.-H. He, B. A. Ovrut, and R. Reinbacher, The spectra of heterotic standard model vacua, JHEP 06 (2005) 070, hep-th/0411156.

[39] D.-E. Diaconescu, B. Florea, S. Kachru, and P. Svrcek, Gauge - mediated supersymmetry breaking in string compactifications, JHEP 02 (2006) 020, hep-th/0512170. 
[40] J.-P. Derendinger and R. Sauser, A five-brane modulus in the effective $n=1$ supergravity of m-theory, Nucl. Phys. B598 (2001) 87-114, hep-th/0009054.

[41] E. Lima, B. A. Ovrut, J. Park, and R. Reinbacher, Non-perturbative superpotential from membrane instantons in heterotic m-theory, Nucl. Phys. B614 (2001) 117-170, hep-th/0101049.

[42] E. Lima, B. A. Ovrut, and J. Park, Five-brane superpotentials in heterotic m-theory, Nucl. Phys. B626 (2002) 113-164, hep-th/0102046.

[43] A. Lukas, B. A. Ovrut, and D. Waldram, On the four-dimensional effective action of strongly coupled heterotic string theory, Nucl. Phys. B532 (1998) 43-82, hep-th/9710208.

[44] P. Candelas and X. de la Ossa, Moduli space of calabi-yau manifolds, Nucl. Phys. B355 (1991) 455-481.

[45] A. Lukas, B. A. Ovrut, and D. Waldram, Non-standard embedding and five-branes in heterotic m- theory, Phys. Rev. D59 (1999) 106005, hep-th/9808101.

[46] E. Buchbinder and B. A. Ovrut, Vector bundle moduli, Russ. Phys. J. 45 (2002) 662-669.

[47] K. Behrndt and S. Gukov, Domain walls and superpotentials from $m$ theory on calabiyau three-folds, Nucl. Phys. B580 (2000) 225-242, hep-th/0001082.

[48] M. Becker and D. Constantin, A note on flux induced superpotentials in string theory, JHEP 08 (2003) 015, hep-th/0210131.

[49] M. Dine, R. Rohm, N. Seiberg, and E. Witten, Gluino condensation in superstring models, Phys. Lett. B156 (1985) 55.

[50] V. S. Kaplunovsky and J. Louis, Model independent analysis of soft terms in effective supergravity and in string theory, Phys. Lett. B306 (1993) 269-275, hep-th/9303040.

[51] A. Brignole, L. E. Ibanez, and C. Munoz, Towards a theory of soft terms for the supersymmetric standard model, Nucl. Phys. B422 (1994) 125-171, hep-ph/9308271.

[52] H. P. Nilles, M. Olechowski, and M. Yamaguchi, Supersymmetry breaking and soft terms in m-theory, Phys. Lett. B415 (1997) 24-30, hep-th/9707143. 
[53] P. Horava, Gluino condensation in strongly coupled heterotic string theory, Phys. Rev. D54 (1996) 7561-7569, hep-th/9608019.

[54] Z. Lalak and S. Thomas, Gaugino condensation, moduli potential and supersymmetry breaking in m-theory models, Nucl. Phys. B515 (1998) 55-72, hep-th/9707223.

[55] A. Lukas, B. A. Ovrut, and D. Waldram, Five-branes and supersymmetry breaking in m-theory, JHEP 04 (1999) 009, hep-th/9901017.

[56] E. Witten, World-sheet corrections via d-instantons, JHEP 02 (2000) 030, hep-th/9907041.

[57] E. I. Buchbinder, R. Donagi, and B. A. Ovrut, Superpotentials for vector bundle moduli, Nucl. Phys. B653 (2003) 400-420, hep-th/0205190.

[58] E. I. Buchbinder, R. Donagi, and B. A. Ovrut, Vector bundle moduli superpotentials in heterotic superstrings and m-theory, JHEP 07 (2002) 066, hep-th/0206203.

[59] E. Buchbinder, R. Donagi, and B. A. Ovrut, Vector bundle moduli and small instanton transitions, JHEP 06 (2002) 054, hep-th/0202084.

[60] C. Beasley and E. Witten, Residues and world-sheet instantons, JHEP 10 (2003) 065, hep-th/0304115.

[61] to appear.

[62] K. Dasgupta, G. Rajesh, and S. Sethi, M theory, orientifolds and g-flux, JHEP 08 (1999) 023, hep-th/9908088.

[63] S. B. Giddings, S. Kachru, and J. Polchinski, Hierarchies from fluxes in string compactifications, Phys. Rev. D66 (2002) 106006, hep-th/0105097.

[64] S. Kachru, M. B. Schulz, and S. Trivedi, Moduli stabilization from fluxes in a simple iib orientifold, JHEP 10 (2003) 007, hep-th/0201028.

[65] S. Kachru, M. B. Schulz, P. K. Tripathy, and S. P. Trivedi, New supersymmetric string compactifications, JHEP 03 (2003) 061, hep-th/0211182.

[66] A. R. Frey and J. Polchinski, $N=3$ warped compactifications, Phys. Rev. D65 (2002) 126009, hep-th/0201029. 
[67] S. Gukov, C. Vafa, and E. Witten, Cft's from calabi-yau four-folds, Nucl. Phys. B584 (2000) 69-108, hep-th/9906070.

[68] T. R. Taylor and C. Vafa, Rr flux on calabi-yau and partial supersymmetry breaking, Phys. Lett. B474 (2000) 130-137, hep-th/9912152.

[69] G. Curio, A. Klemm, D. Lust, and S. Theisen, On the vacuum structure of type ii string compactifications on calabi-yau spaces with h-fluxes, Nucl. Phys. B609 (2001) 3-45, hep-th/0012213.

[70] G. Curio and A. Krause, Enlarging the parameter space of heterotic m-theory flux compactifications to phenomenological viability, Nucl. Phys. B693 (2004) 195-222, hep-th/0308202.

[71] V. Balasubramanian and P. Berglund, Stringy corrections to kaehler potentials, susy breaking, and the cosmological constant problem, JHEP 11 (2004) 085, hep-th/0408054.

[72] V. Balasubramanian, P. Berglund, J. P. Conlon, and F. Quevedo, Systematics of moduli stabilisation in calabi-yau flux compactifications, JHEP 03 (2005) 007, hep-th/0502058.

[73] S. Weinberg, Anthropic bound on the cosmological constant, Phys. Rev. Lett. 59 (1987) 2607.

[74] K. Choi, A. Falkowski, H. P. Nilles, M. Olechowski, and S. Pokorski, Stability of flux compactifications and the pattern of supersymmetry breaking, JHEP 11 (2004) 076, hep-th/0411066.

[75] K. Choi, A. Falkowski, H. P. Nilles, and M. Olechowski, Soft supersymmetry breaking in kklt flux compactification, Nucl. Phys. B718 (2005) 113-133, hep-th/0503216. 